\title{
Extracting Rules via Markov Chains for Cryptocurrencies Returns Forecasting
}

\author{
Kerolly Kedma Felix do Nascimento ${ }^{1}$ (D) - Fábio Sandro dos Santos ${ }^{1}$ • \\ Jader Silva Jale ${ }^{1} \cdot$ Silvio Fernando Alves Xavier Júnior ${ }^{2} \cdot$ Tiago A. E. Ferreira $^{1}$
}

Accepted: 17 January 2022

(C) The Author(s), under exclusive licence to Springer Science+Business Media, LLC, part of Springer Nature 2022

\begin{abstract}
With the growing popularity of digital currencies known as cryptocurrencies, there is a need to develop models capable of robustly analyzing and predicting the value of future returns in these markets. In this article, we extract behavior rules to predict the values of future returns in the Bitcoin, Ethereum, Litecoin, and Ripple closing series. We used categorical data in the analyses and Markov chain models from the first to the tenth order to propose a new way of establishing possible future scenarios, in which we analyze the dependence of memory on the dynamics of the process. We used the measurements of accuracy Mean Quadratic Error, Absolute Error Mean Percentage, and Absolute Standard Deviation for the choice of the best models. Our findings reveal that cryptocurrencies have long-range memory. Bitcoin, Ethereum, and Ripple exposed seven steps of memory, while Litecoin displayed nine memory steps. From the transitions between states that happened the most, we defined decision rules that assisted in the definition of future returns in the series. Our results can support the decisions of traders, investors, crypto-traders, and policy-makers.
\end{abstract}

Keywords Digital market · Rule support - Granularity · Time series forecasting · Markov chains · Long-range memory

Kerolly Kedma Felix do Nascimento

kerollyfn@gmail.com

Tiago A. E. Ferreira

tiago.espinola@ufrpe.br

1 Department of Statistics and Informatics, Federal Rural University of Pernambuco, Recife, Pernambuco, Brazil

2 Department of Statistics, State University of Paraíba, Campina Grande, Paraíba, Brazil 


\section{Introduction}

Recently, the cryptocurrency market has become popular among investors due the technology known as blockchain allows the distribution of data and records among market participants based on cryptographic protection (Stosic et al. 2019). There are currently more than 2000 cryptocurrencies on the market (CoinMarketCap 2020a; Cheng et al. 2019), the most popular being Bitcoin (BTC), first introduced in 2008 and with the largest market capitalization (Song et al. 2019). In the ranking of the top 100 cryptocurrencies, the four more important are Bitcoin (often in the first position), Ethereum (ETH), Litecoin (LTC), and Ripple (XRP). These four cryptocurrencies are considered the largest in the digital market. They account for about $80 \%$ of all cryptocurrency market capitalization and are often the focus of investigation in academic studies (CoinMarketCap 2020b).

In this perspective, several researchers have endeavored to understand the dynamics of cryptocurrency time series better. Different approaches have been adopted with the aid of computational tools. For example, the multifractality techniques were employed to analyze the Bitcoin market (Takaishi 2018; Mensi et al. 2019; El Alaoui et al. 2019). It is also possible to find studies with the modeling of volatilities of some cryptocurrencies (Fakhfekh and Jeribi 2020; Yi et al. 2018; Phillip et al. 2019), and the use of a hybrid structure for forecasting volatility (Almeida et al. 2015; Bouri et al. 2019; Peng et al. 2018). Caporale et al. (2018) investigated the persistence in the time series of Bitcoin, Litecoin, Ripple, and Dash cryptocurrencies. The study results revealed a positive correlation between current and past values, with changes over time. Also, one can see the inefficiency of the studied markets, ensuring a certain degree of predictability into the series. Catania and Grassi (2017) analyzed more than 600 cryptocurrencies. They emphasized the main four (BTC, ETH, LTC, and XRP) and produced a dynamic model with the ability to use asymmetries and long memory in volatility; its forecast results were promising with the use of a robust filter where there is a variable asymmetry over time.

Bouri et al. (2021b) analyzed the dynamic equicorrelation between twelve cryptocurrencies from 2015 to 2019. The authors noted that if Bitcoin, for example, suffers a serious failure, other cryptocurrencies may be affected. In addition, evidence suggests a time-varying mean return equicorrelation. It also points out that the integration of markets has its causes in the volume of business and measures of uncertainty. Schinckus et al. (2021) studied 1636 cryptocurrencies and observed densification of interconnections between virtual currencies. The authors suggested that cryptocurrencies presented short-term interdependence between them. The power of influence of each cryptocurrency varied year after year. Schinckus et al. (2020) related Bitcoin trading volume data to energy consumption to verify the impact on climate change. Their results revealed a significant and increasing positive influence between energy consumption and cryptocurrency activities. They also verified that the increase in energy consumption is linked to the increase in cryptocurrency activities. Naeem et al. (2021) used Multifractal Detrended Fluctuation Analysis (MF-DFA) to assess the asymmetric efficiency of the Bitcoin, 
Ethereum, Litecoin and Ripple series during the COVID-19 pandemic. The approach revealed that in downtrends, there is weaker multifractality than in uptrends. The two largest cryptocurrencies (Bitcoin and Ethereum) were the most affected. They presented a rapid recovery from the slide into inefficiency during the pandemic period. Bouri et al. (2021a) investigated seven cryptocurrencies between 2015 and 2020 and observed instability of the connectivity system under extreme events, such as COVID-19. The authors pointed out besides Bitcoin, other cryptocurrencies such as Ethereum contribute to the stability of the connection network in the cryptocurrency market. This fact indicates the importance of other cryptocurrencies, in addition to Bitcoin, must also be investigated during monitoring.

Regarding forecasting, Markov chain models have been used for various types of phenomena, such as wind speed predictability (Song et al. 2014), the stock market (Kiral and Uzun 2017), water resources management (Rezaeianzadeh et al. 2016), and inflammatory breast cancer with bone metastasis (Fujii et al. 2019). Leaning on forecasting financial markets using Markov chains, Svoboda and Lukas (2012) used four Markov chain models to forecast trends in Prague stock indexes and analyze investment strategies. The authors used discrete state spaces to define the models and generate the transition probability matrices used in the forecast. Soloviev et al. (2011) performed the forecast with Markov chains for American, European, and Asian stock indexes. The results presented in the study are very favorable and reveal the efficiency of the model. There are several works on cryptocurrency forecasting in the literature (Sun et al. 2020; Chowdhury et al. 2019, 2020; Velankar et al. 2018; Catania et al. 2019) and a variety of Markov chain applications for forecasting different phenomena (Zhan-Li and Jin-Hua 2011; Liu et al. 2009; Chaudhuri et al. 2014; Carvalho and Moura 2015). However, our study is the first study that uses Markov chains from the first to the tenth order to extract rules to be used to forecast cryptocurrency returns.

Namely, the advantages of our approach lie in the development of a simple model that is easy to use, understand and implement. We verified the stochastic dependence of the first to tenth-order models of the Markov chains, analyzing the adjustment errors and investigating the existence of dependence on higher orders in the process. About the disadvantages, the dependence was considered by directly relating the observations with each order of the Markov chains. Furthermore, as we increase the order of the Markov model, there is an increase in memory consumption, leading to a memory cost of $\Theta\left(n^{m+1}\right)$, i.e. the memory cost increases with the number of state transition $n$ raised to power $m+1$, where $m$ is the Markov chains order. In this way, this prevents us from using higher orders in the models. In this sense, our observations are restricted only to the investigated orders, leading to statements regarding only those orders.

Therefore, in this paper, the main contributions are as follows: extract rules from the dynamics of cryptocurrencies return time series via Markov Chains to determine possible future scenarios, analyzing memory dependence on the process dynamics. The first to tenth order models were used to assess which one provides the slightest 
forecast error for the time series. We use time series data for the four main cryptocurrencies by market capitalization: Bitcoin, Ethereum, Litecoin e Ripple.

The rest of this paper is structured as follows: Sect. 2 presents a brief description of Markov chain models. Section 3 displays the evaluation metrics used, Sect. 4 describes the investigated data set. Sections 5 and 6 expose the data pre-processing, the experiment protocol, the empirical results, and analysis, respectively. Finally, Sect. 7 sets out the main conclusions.

\section{Markov Chain}

Markov chain is a type of stochastic process in which the result of the next state of an experiment depends only on the result of the experiment in the current state (Kemeny and Snell 1976).

Let be a stochastic process of a random variable $X$. The data are discretized and assigned to a state $S$ in the set $\{1,2, \ldots, n\}$. Since $n$ is the number of states in the system, the transition probability matrix $(n \times n)$ is defined as (Sahin and Sen 2001),

$$
P_{t, t+1}=\left[\begin{array}{ccccccc}
p_{11} & p_{12} & p_{13} & p_{14} & p_{15} & \cdots & p_{1 n} \\
p_{21} & p_{22} & p_{23} & p_{24} & p_{25} & \cdots & p_{2 n} \\
p_{31} & p_{32} & p_{33} & p_{34} & p_{35} & \cdots & p_{3 n} \\
p_{41} & p_{42} & p_{43} & p_{44} & p_{45} & \cdots & p_{4 n} \\
\vdots & \vdots & \vdots & \vdots & \vdots & \cdots & \vdots \\
p_{n 1} & p_{n 2} & p_{n 3} & p_{n 4} & p_{n 5} & \cdots & p_{n n}
\end{array}\right]
$$

wherein $p_{i j}$ represents the probability of transition from state $i$ at time $t$ to state $j$ at time $t+1 . p_{i j}$ is a conditional probability constant over time and can be written as $\operatorname{Pr}\left(X_{t}=j \mid X_{t-1}=i\right)=p_{i j}$. Two properties are always valid (da Silva et al. 2019): ( $i$ ) $0 \leq p_{i j} \leq 1$ and (ii) $\sum_{j=1}^{n} p_{i j}=1$. Through the number of transitions from state $i$ to the state $j(i \rightarrow j)$, we obtain the maximum likelihood estimator corresponding to the inputs of the matrix (Papaefthymiou and Klockl 2008),

$$
p_{i j}=\frac{n_{i j}}{\sum_{j} n_{i j}},
$$

where $n_{i j}$ indicates the total number of times that the $i \rightarrow j$ transition occurred in the transition probability matrix.

The probability of transition to the next state, which is dependent on the current state and the previous state $\left(p_{i j k}\right)$, makes up the second-order transition probability matrix expressed as (Shamshad et al. 2005), 


$$
P=\left[\begin{array}{ccccccc}
p_{111} & p_{112} & p_{113} & p_{114} & p_{115} & \cdots & p_{11 k} \\
p_{121} & p_{122} & p_{123} & p_{124} & p_{125} & \cdots & p_{12 k} \\
p_{131} & p_{132} & p_{133} & p_{134} & p_{135} & \cdots & p_{1, k} \\
\vdots & \vdots & \vdots & \vdots & \vdots & \cdots & \vdots \\
p_{1 k 1} & p_{1 k 2} & p_{1 k 3} & p_{1 k 4} & p_{1 k 5} & \cdots & p_{1 k k} \\
p_{211} & p_{212} & p_{213} & p_{214} & p_{215} & \cdots & p_{21 k} \\
p_{221} & p_{222} & p_{223} & p_{224} & p_{225} & \cdots & p_{22 k} \\
p_{231} & p_{232} & p_{233} & p_{234} & p_{235} & \cdots & p_{23 k} \\
\vdots & \vdots & \vdots & \vdots & \vdots & \cdots & \vdots \\
p_{k k 1} & p_{k k 2} & p_{k k 3} & p_{k k 4} & p_{k k 5} & \cdots & p_{k k k}
\end{array}\right]
$$

where $p_{i j k}$ represents the probability of the next state $k$, if $j$ represents the current state and $i$ the previous state. The accumulated probability is given by,

$$
P_{i j k}=\sum_{l=1}^{k} p_{i j l},
$$

in which $p_{i j l}$ is the probability of transition on the $i j$-th row in the $k$-th state.

For a Markov Chain matrix of order $m$, future processes are dependent on the past $m$ states. Since $C_{t}$ is a sequence of states, the probabilities can be written as (Ky and Tuyen 2018):

$$
\operatorname{Pr}\left(C_{t+1} \mid C_{t}, C_{t-1}, \ldots, C_{1}\right)=\operatorname{Pr}\left(C_{t+1} \mid C_{t}, C_{t-1}, \ldots, C_{t-m+1}\right) .
$$

Thus, the transition probability matrix for a Markov Chain with memory $m$ is given by (Guo and Ching 2021):

$$
P=\left[\begin{array}{cccccc}
p_{11 \ldots 111} & p_{11 \ldots 112} & p_{11 \ldots 113} & \cdots & p_{11 \ldots 11 m-1} & p_{11 \ldots 11 m} \\
p_{11 \ldots 121} & p_{11 \ldots 122} & p_{11 \ldots 123} & \cdots & p_{11 \ldots 12 m-1} & p_{11 \ldots 12 m} \\
\vdots & \vdots & \vdots & \ldots & \vdots & \vdots \\
p_{11 \ldots 1 m 1} & p_{11 \ldots 1 m 2} & p_{11 \ldots 1 m 3} & \cdots & p_{11 \ldots 1 m m-1} & p_{11 \ldots 1 m m} \\
p_{11 \ldots 211} & p_{11 \ldots 212} & p_{11 \ldots 213} & \cdots & p_{11 \ldots 21 m-1} & p_{11 \ldots 21 m} \\
p_{11 \ldots 221} & p_{11 \ldots 222} & p_{11 \ldots 223} & \cdots & p_{11 \ldots 2 m-1} & p_{11 \ldots 22 m} \\
\vdots & \vdots & \vdots & \cdots & \vdots & \vdots \\
p_{11 \ldots 2 m 1} & p_{11 \ldots 2 m 2} & p_{11 \ldots 2 m 3} & \cdots & p_{11 \ldots 2 m, m-1} & p_{11 \ldots 2 m m} \\
\vdots & \vdots & \vdots & \cdots & \vdots & \vdots \\
p_{m m \ldots m 11} & p_{m m \ldots m 12} & p_{m m \ldots m 13} & \cdots & p_{m m m m 1 m-1} & p_{m m \ldots m 1 m} \\
p_{m m \ldots m 21} & p_{m m \ldots m 22} & p_{m m \ldots m 23} & \cdots & p_{m m m m 2 m-1} & p_{m m \ldots m 2 m} \\
\vdots & \vdots & \vdots & \cdots & \vdots & \vdots \\
p_{m m \ldots m m 1} & p_{m m \ldots m m 2} & p_{m m \ldots m m 3} & \cdots & p_{m m \ldots m m-1} & p_{m m \ldots m m} \ldots m
\end{array}\right]
$$




\section{Evaluation Metrics}

\subsection{Jarque-Bera Test}

The bilateral Jarque-Bera test was initially proposed in 1987 (Jarque and Bera 1987) to verify the null hypothesis that the sample observations follow a normal distribution with unknown mean and variance. The Jarque-Bera test uses kurtosis and asymmetry coefficients as parameters as an alternative to the Pearson distribution system (Zhang et al. 2018). The Jarque-Bera test statistic is given by,

$$
J B=\frac{N}{6}\left(s^{2}+\frac{(k-3)^{2}}{4}\right)
$$

where $N$ is the number of observations, $s$ and $k$ are the asymmetry and kurtosis coefficients of the observations. When the sample is considerably large, the JB statistic follows a Chi-Square distribution with two degrees of freedom: $J B \sim \chi_{2}^{2}$.

\subsection{Accuracy Measures}

Hyndman and Koehler (2006) state that accuracy measures such as Root Mean Square Error (RMSE) and Mean Absolute Error (MAE) should be used to compare databases with equal scales and that RMSE has a higher sensitivity to outliers than the MAE. On the other hand, accuracy measures based on percentage errors, such as Mean Absolute Percent Error (MAPE), do not depend on the scale of the data sets and may be used in comparisons with different databases. In this sense, to identify the matrix order that provides the least error in the generation of cryptocurrency forecasts, we used the RMSE, MAE, and MAPE accuracy measures. Table 1 exhibits the mathematical expressions of each measure applied (Ferreira et al. 2008; Guo et al. 2018; Antulov-Fantulin et al. 2020).

Where $x_{i}$ represents each observation in the series of cryptocurrencies returns, $\hat{x_{i}}$ the estimate of the observations in the analyzed series, and $N$ the number of time series points. For all the performance measures employed, the best performance is obtained when they have the lowest value (Altan et al. 2019). Therefore, with the choice of the best order of the Markov chain model, more significant the accuracy estimate and better the model's suitability for the data forecast.

\subsection{Rule and Support}

In this work, we will understand the rule as the information obtained from the $i \rightarrow j$ transitions of the Markov Chains transition probability matrices analyzed. The rule support is a percentage statistical measure, which reflects the proportion of values that meet the antecedent $(i)$ and the consequent $(j)$ of the rule (Mata et al. 2002). The application of this measure aims to provide aid to decision making regarding the future scenarios generated. Mathematically, let $D$ be the database that contains all possible transactions $(T)$, that is, $T \in D$. A rule $A \rightarrow B$ reveals that if a transaction contains items $a \in A$, it probably also contains items $b \in B$. Support for $A$ is 
provided by $\operatorname{supp}_{D}(A)=|\{T \in D \mid A \subseteq T\}| /|D|$. Thus, support to the rule is determined by (Hipp et al. 2002; Han et al. 2011):

$$
\operatorname{supp}_{D}(A \rightarrow B)=\operatorname{supp}_{D}(A \cup B)=P(A \cup B) .
$$

\section{Datasets}

We use four times series of highly liquid cryptocurrencies in the market: Bitcoin, Ethereum, Litecoin, and Ripple. The databases were composed of daily closing prices in US dollars from October 1st, 2016 to November 30th, 2019 (1156 observations for each cryptocurrency), available at https:/coinmetrics.io/ datadownloads/. Fig. 1 illustrates the price series of the four cryptocurrencies over the period investigated. Between December 2017 and March 2018 occurred the highest price increases for all cryptocurrencies studied. For Bitcoin, we observed that from June to December 2019, although the values are up compared to the previous semester, they appear to be in a downward trend.

\section{Data Pre-processing and Protocol of Experiments}

We calculate the series of logarithmic price returns (see Fig. 2) using the equation $r_{t}=\log \left(z_{t}\right)-\log \left(z_{t-1}\right)$, where $z_{t}$ corresponds to the observed value for the current day and $z_{t-1}$ the observed value for the previous day, that is, one step back. We note that returns show high variability over the entire period, with higher peaks in 2017 and the first quarter of 2018.

The database utilized for each cryptocurrency was separated into two sets: i) $95 \%$ for training (from the first observations), and ii) the last 5\% for testing. Table 2 presents the descriptive statistics of the series of complete returns, training and testing. The average values of the returns presented negative values only for the test series. The standard deviation values indicate similar volatilities in the series of returns. The kurtosis of these cryptocurrencies can assist in checking for volatility. We observed high kurtosis values for some cryptocurrencies, such as Litecoin in the complete series (14.5200) and training set (14.2462) and Ripple also in the complete series (14.9041) and training set (14.3897).

In contrast, the other cryptocurrencies had kurtosis values $<3$. In particular, the lowest kurtosis was found for Ripple in the test series (0.787) and with a very low

Table 1 Statistical accuracy measures used for the forecast comparison

\begin{tabular}{ll}
\hline Statistics & Mathematical equation \\
\hline MAPE & $\frac{1}{N} \sum_{i=1}^{N}\left|\frac{x_{i}-\hat{x_{i}}}{x_{i}}\right| \times 100 \%$ \\
MAE & $\frac{1}{N} \sum_{i=1}^{N}\left|x_{i}-\hat{x_{i}}\right|$ \\
RMSE & {$\left[\frac{1}{N} \sum_{i=1}^{N}\left(x_{i}-\hat{x_{i}}\right)^{2}\right]^{\frac{1}{2}}$} \\
\hline
\end{tabular}




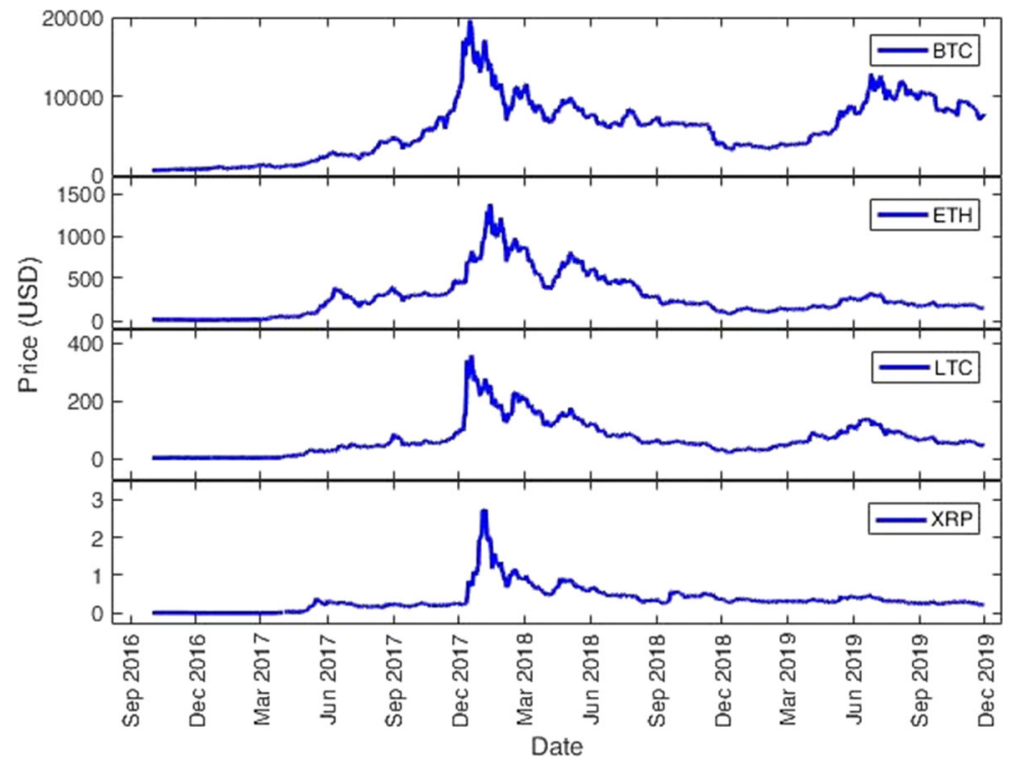

Fig. 1 The plot of Bitcoin, Ethereum, Litecoin and Ripple price observation series

value when compared to the Normal distribution (kurtosis=3), reflecting a platicurtic curve of the data.

Asymmetry values other than zero can reveal the incidence of profit and loss probabilities based on series of returns. Positive asymmetry values indicate values

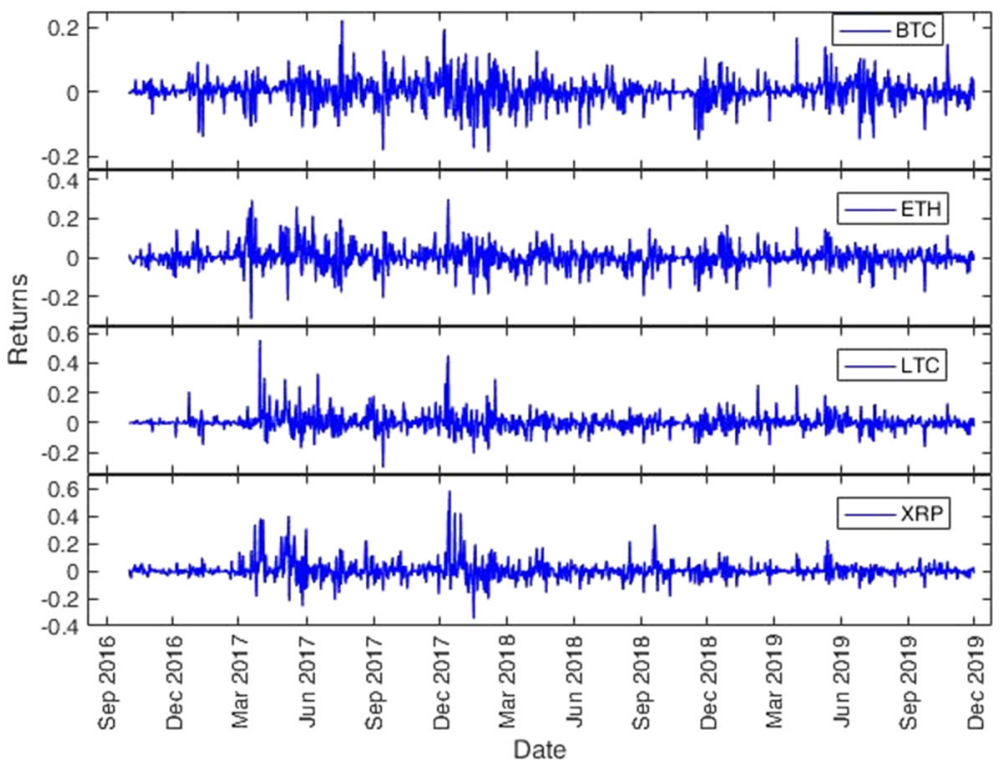

Fig. 2 The plot of the Bitcoin, Ethereum, Litecoin and Ripple return series 
above the average of the normal distribution, making the tail heavier on the right side of the distributions. However, negative asymmetry values reveal a greater probability of lower values than the average of the normal distribution, i.e., the tail of the data distribution is located on the left side of the average. In this paper, the asymmetry values were positive (except for Bitcoin in the complete-return series and training series), implying a higher probability of positive returns. The JarqueBera (JB) test, performed to identify whether the data follows a normal distribution, revealed a significant difference between the $p$-value of all BTC, ETH, LTC, and XRP series, except for the XRP test series. In this way, it means that the data in these series do not come from a normal distribution and corroborates with characteristics found in the financial markets (Bouri et al. 2019; Wu et al. 2018).

For the analysis, it is necessary to categorize the series. The data granularity can influence the forecast values generated. The return times series amplitudes typically are in order of some decimals (see Table 2). In this way, we defined four distinct granularities $(0.1,0.01,0.001$, and 0.0001$)$ to find the best one for a given time series. The choice of these granularitiy values is just a simple form to variate the discretization of the return times series, where the smaller the granularity, the more precise the state discretization. However, it could make different choices for these granularities, where different categorization methodologies will be research themes in future works. These granularities are the range of the categories where the time series data will be classified. The entire categorization range is defined by the minimum (min) and maximum $(\max )$ values of the time series, subtracting and adding a granular value,

- Granularity 0.1: [min - 0.1, $\max +0.1$ [, with subintervals varying from 0.1 to 0.1 ;

Table 2 Descriptive statistics and Jarque-Bera test of the series of returns, for the complete series, of training and testing of the four cryptocurrencies

\begin{tabular}{llllllllll}
\hline & Returns & Min. & Mean & SD & Max. & kurt. & Asym. & JB & $p$-value \\
\hline BTC & Complete & -0.1861 & 0.0022 & 0.0424 & 0.2241 & 6.1795 & -0.0517 & 487.01 & $2.2 \mathrm{e}-16$ \\
& Trainning & -0.1861 & 0.0024 & 0.0428 & 0.2241 & 6.1098 & -0.0953 & 443.71 & $2.2 \mathrm{e}-16$ \\
& Test & -0.0738 & -0.0015 & 0.0341 & 0.1495 & 8.3052 & 1.4335 & 87.883 & $2.2 \mathrm{e}-16$ \\
ETH & Complete & -0.3138 & 0.0021 & 0.0578 & 0.3006 & 6.8624 & 0.3041 & 735.76 & $2.2 \mathrm{e}-16$ \\
& Trainning & -0.3138 & 0.0024 & 0.0588 & 0.3006 & 6.7190 & 0.2914 & 647.72 & $2.2 \mathrm{e}-16$ \\
& Test & -0.0817 & -0.0025 & 0.0341 & 0.1178 & 5.0719 & 0.4620 & 12.437 & 0.001992 \\
LTC & Complete & -0.3040 & 0.0022 & 0.0625 & 0.5568 & 14.5200 & 1.6440 & 6906.9 & $2.2 \mathrm{e}-16$ \\
& Trainning & -0.3040 & 0.0025 & 0.0635 & 0.5568 & 14.2462 & 1.6335 & 6268.9 & $2.2 \mathrm{e}-16$ \\
& Test & -0.0848 & -0.0031 & 0.0374 & 0.1328 & 4.8557 & 0.4365 & 10.164 & 0.006208 \\
XRP & Complete & -0.3477 & 0.0029 & 0.0704 & 0.5829 & 14.9041 & 1.9007 & 7508.6 & $2.2 \mathrm{e}-16$ \\
& Trainning & -0.3477 & 0.0031 & 0.0719 & 0.5829 & 14.3897 & 1.8688 & 6578.2 & $2.2 \mathrm{e}-16$ \\
& Test & -0.0663 & -0.0016 & 0.0301 & 0.0842 & 3.8971 & 0.3516 & 34.456 & 0.1786 \\
\hline
\end{tabular}


- Granularity 0.01 : [min $-0.01, \max +0.01$ [, with subintervals varying from 0.01 to 0.01 ;

- Granularity 0.001: [min $-0.001, \max +0.001[$, with subintervals varying from 0.001 to 0.001 ;

- Granularity 0.0001: [min - 0.0001, $\max +0.0001[$, with subintervals varying from 0.0001 to 0.0001 .

The historical data series was used to define the transition probability matrix states and generate the forecast values with the obtained matrices. We use Eqs. 1, 2, and 3 to define transition probability matrices in Markov Chain models. The RMSE, MAPE, and MAE errors were used to inspect which order in the Markov chain had the smallest error measures in the predictions. Based on the best models obtained for each cryptocurrency series, we analyzed the rules that presented the "best support" in the transitions between states with the help of Eq. 5. We defined the probable return intervals in future scenarios for Bitcoin, Ethereum, Litecoin, and Ripple.

\section{Empirical Results and Analysis}

With the series categorized, we define the states of the transition matrices of the values so that the total number of states for each granularity is the total number of categories minus one. For example, for BTC at granularity 0.1 , we have the limit for categorization [ $-0.286095194,0.324052773[$. Here, it was generated six categories and five possible states of the transition matrices. Subsequently, we calculated the transition matrices from the 1st to 10th order of the Markov Chain. All Markov models were employed for each granularity of the four cryptocurrencies studied. Fig. 3 shows the transition probability matrices between the states of the Markov Chain model for the 1st order at granularity 0.1 for all the cryptocurrencies studied.

One can note that in this granularity, except for ETH, in each matrix obtained, there is at least one state in which the transition probability is equal to 1. For example, for the BTC transition matrix of order 1, the probability of transition from state $5 \rightarrow 2$ is 1 . In this way, it means that if in an instant $t$, the categorical return of BTC is in state 5 , in instant $t+1$, it will be in state 2. Likewise, this happens for the $8 \rightarrow 2$ and $9 \rightarrow 3$ transitions in LTC and $1 \rightarrow 5$ and $10 \rightarrow 3$ in XRP. Nevertheless, we found that for ETH the greatest probabilities identified occurred in the transitions $3 \rightarrow 4$ (0.6413), $2 \rightarrow 4$ (0.6207) and $4 \rightarrow 4$ (0.6076).

Applying the transition probabilities among the states, we generate future values for the series with the ten Markov Chain models. According to the criteria mentioned in Section 3, the RMSE and MAE error measures indicate the orders of the Markov Chain models that provide better accuracy in the values generated within each granularity. On the other hand, the MAPE error measure provides information for comparing granularities. Table 3 shows the values of the RMSE, MAE, and MAPE accuracy measures obtained when comparing the future values generated and the values of the test series for the best models obtained. At each granularity, we observe the cryptocurrencies individually. Using the RMSE and MAE measures, we compare the predictions for the ten Markov chain models 
applied. The Markov chain models that we see in Table 3 are the ones that showed the smallest errors in the comparisons.

There was a significant reduction in the values from the first $(0.1)$ to the second (0.01) granularities regarding the MAPE measure. At the third granularity (0.001), errors increased considerably. Note that for ETH, LTC, and XRP, the MAPE values are approximately three times greater than the second granularity. For the fourth granularity (0.0001), the errors were even greater. Thus, the MAPE measure revealed that the predictions with the Markov models at 0.01 granularity provided the best results. This result indicates that this is the ideal granularity to proceed with the analyzes. At 0.01 granularity, we observed that the predictions with Markov of order 7 provided better results for BTC, ETH, and XRP, indicating that these series had seven memory steps, that is, to know the current state $i$, seven states must be considered previous $(i-7)$. On the other hand, to define the LTC status $i$, it is necessary to use information from the state $(i-9)$, according to the Markov model of order 9 .
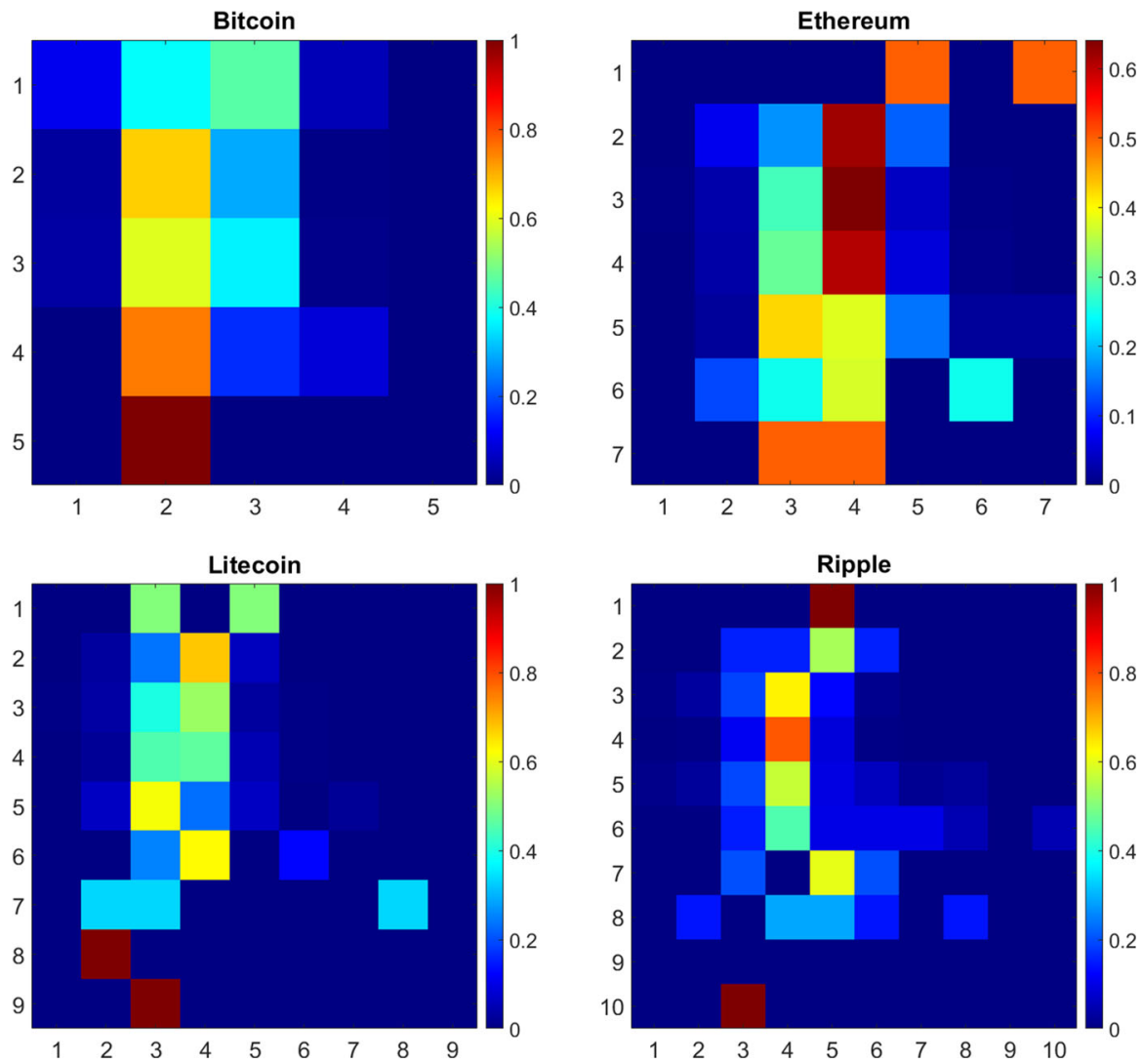

Fig. 3 Heatmaps of the transition probability matrices for Markov chains of first order of Bitcoin, Ethereum, Litecoin and Ripple at 0.1 granularity 
In the literature, there is divergence regarding the existence of memory in the series of cryptocurrency markets (Rambaccussing and Mazibas 2020). For example, Tiwari et al. (2018) and Soylu et al. (2020) oppose the existence of long memory in the series of returns. Stosic et al. (2019) claim that there is no correlation in the prices of the top 50 cryptocurrencies (including Bitcoin, Ethereum, Litecoin and Ripple) calculated by logarithmic returns, implying greater efficiency in these markets. In contrast, Jiang et al. (2018), Zhang et al. (2018) and Charfeddine and Maouchi (2019) find evidence of long memory in the series of cryptocurrency returns. In our study, besides finding evidence of the presence of long-range memory in the series, we identified how many memory steps each has. Results show that Litecoin is the cryptocurrency that presents the longest period of dependence, with nine steps of memory, while the other cryptocurrencies exhibited seven steps of historical dependence. Figs. 4, 5, 6 and 7 display the forecasts with smallest errors relative to the actual values for each cryptocurrency investigated. These results corroborate with those previously found by Karakoyun and Cibikdiken (2018) when forecasting the next 30 days for the Bitcoin price series using an ARIMA model and also by Altan et al. (2019) for forecasting Litecoin prices using long short-term memory (LSTM), for example.

Examining the best model found for each cryptocurrency investigated, we count the quantities of transitions that occurred among the states, and then we order from the lowest to the highest value. Then, we cut the quantile $75 \%$ of the values found to assess the rule that most happened within each case, that is, to analyze the most recurring transitions. We calculated the transition support of those states that

Table 3 Accuracy of statistical measures for cryptocurrency forecasts

\begin{tabular}{llllll}
\hline Granularity & Criptocurrency & Markov Model & RMSE & MAE & MAPE \\
\hline 0.1 & BTC & 8th-order & 0.767 & $6.061 \mathrm{e}-01$ & 18.617 \\
& ETH & 9th-order & 0.745 & $5.877 \mathrm{e}-01$ & 12.810 \\
& LTC & 9th-order & 0.852 & $7.242 \mathrm{e}-01$ & 16.142 \\
0.01 & XRP & 1st-order & 0.805 & $6.109 \mathrm{e}-01$ & 12.055 \\
& BTC & 7th-order & 3.604 & $2.296 \mathrm{e}+00$ & 11.728 \\
& ETH & 7th-order & 3.975 & $2.774 \mathrm{e}+00$ & 8.646 \\
& LTC & 9th-order & 3.618 & $2.712 \mathrm{e}+00$ & 8.648 \\
0.001 & XRP & 7th-order & 3.481 & $2.513 \mathrm{e}+00$ & 6.908 \\
& BTC & 9th-order & 49.656 & $3.103 \mathrm{e}+01$ & 16.516 \\
& ETH & 2nd-order & 127.909 & $7.859 \mathrm{e}+01$ & 24.255 \\
& LTC & 9th-order & 102.344 & $6.760 \mathrm{e}+01$ & 22.407 \\
0.0001 & XRP & 3rd-order & 115.007 & $5.917 \mathrm{e}+01$ & 17.328 \\
& BTC & 8th-order & 1131.592 & $8.442 \mathrm{e}+02$ & 47.163 \\
& ETH & 9th-order & 1779.246 & $1.166 \mathrm{e}+03$ & 38.395 \\
& LTC & 3rd-order & 1674.517 & $1.158 \mathrm{e}+03$ & 38.471 \\
& XRP & 4th-order & 2201.324 & $1.559 \mathrm{e}+03$ & 46.279 \\
\hline
\end{tabular}


occurred the most to obtain the percentage of transitions that contain the states $\mathrm{X}$ and $\mathrm{Y}$, indicating the relevance of the rule. Table 4 shows the supports obtained for the transitions between states that most happened in our models and their respective transition probabilities.

When looking at Bitcoin, for example, the rule that most occurred was the $19 \rightarrow 19$ transition $(60.34 \%$ of cases). This transition has a 0.26 probability of occurrence. Through Table 4, state 19 corresponds to the return interval from -0.0161 to -0.0061 . Thus, with a transition probability of 0.26 , for a given time $t$, if Bitcoin's return is in the range of -0.0161 to -0.0061 , at time $t+7$ it will remain in the same range with an associated 11.78 MAPE error. Similarly, we conclude that for Ethereum and Ripple at time $t+7$, their returns will remain in the ranges of -0.0138 to -0.0038 and -0.0277 to -0.0177 , with transition probabilities of 0.17 , and 0.16, and MAPE error of 8.65, and 6.91, respectively. For Litecoin, in the instant $t+9$ the return value can migrate from -0.0140 to -0.0040 , to -0.0238 to -0.0140 , or from -0.0238 to -0.0140 , from -0.0140 to -0.0040 as they had equal support value $(36.21 \%$ ) in the rules, both with a transition probability of 0.18 , and MAPE error of 8.65 .

As already mentioned, in this work, we estimated the amount of memory existing in the series of categorized returns of cryptocurrencies. So far, in the literature, studies about memory dependence estimative on cryptocurrency have not been found. This fact makes it impossible to compare our work directly. Works claim there is memory in the series, and others claim there is no memory. We corroborate with the works that claim there is memory in the series and, in addition to this corroboration, we defined the amount of memory existing for the investigated cryptocurrencies.

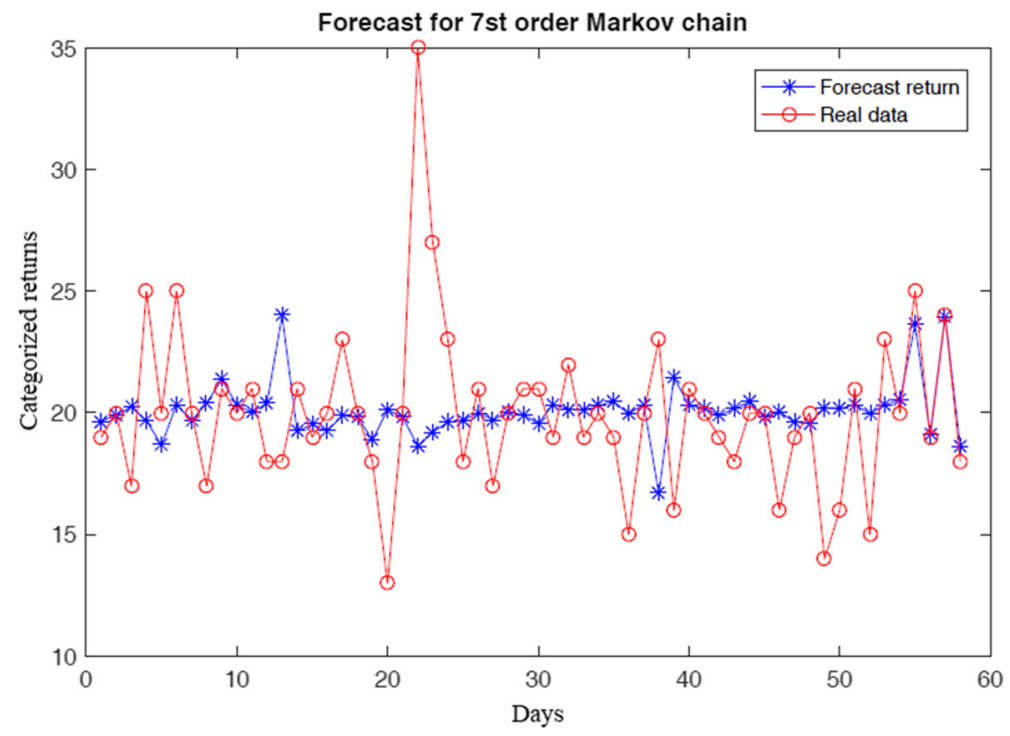

Fig. 4 Forecasts generated for Bitcoin 


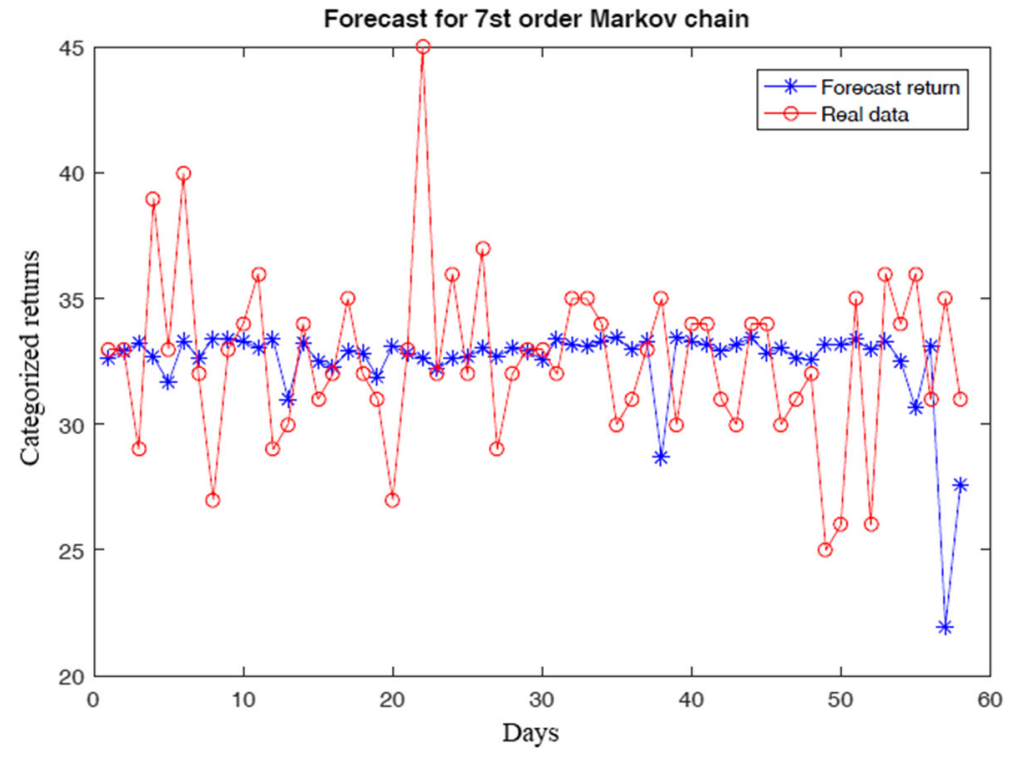

Fig. 5 Forecasts generated for Ethereum

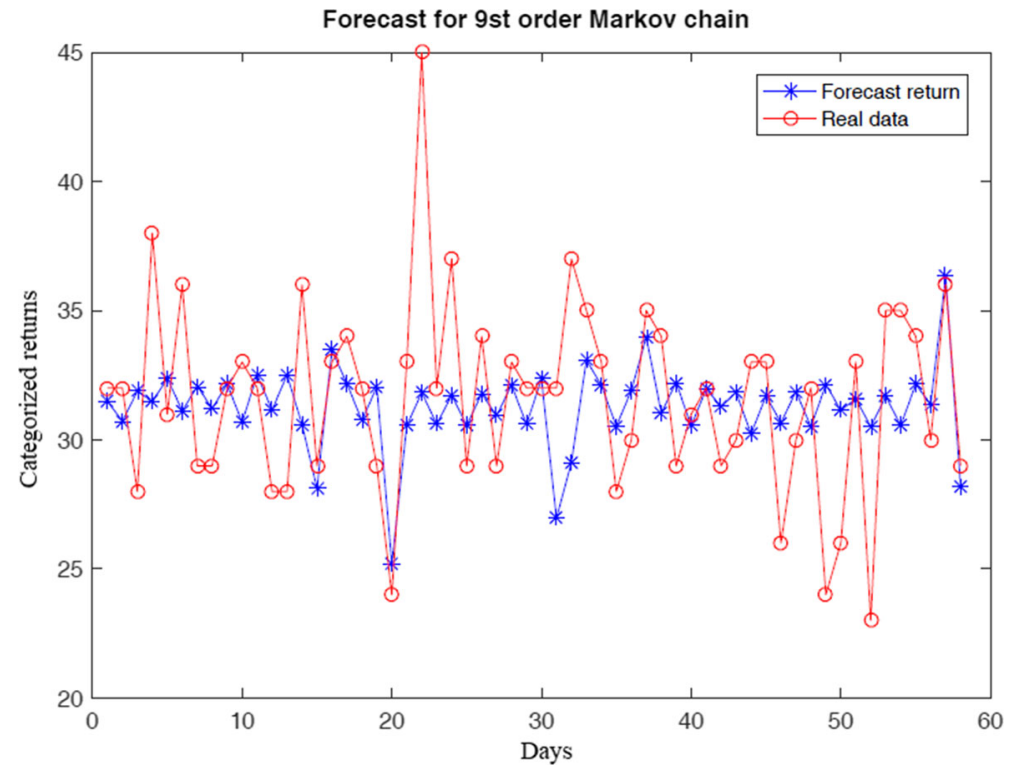

Fig. 6 Forecasts generated for Litecoin 


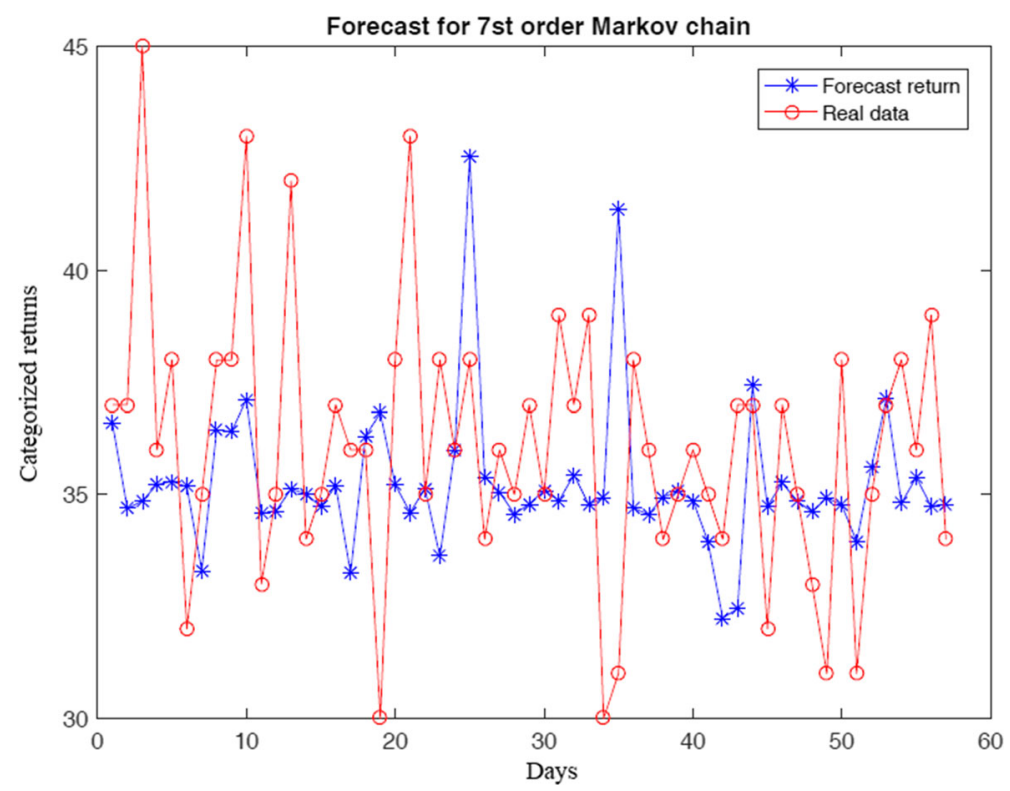

Fig. 7 Forecasts generated for Ripple

\section{Conclusions}

In this article, we use the high-order Markov chain models for the categorized returns series of Bitcoin, Ethereum, Litecoin and Ripple cryptocurrencies to extract forecasting rules. The models were applied in the series with granularity $0.1,0.01$, 0.001 and 0.0001 . According to the RMSE and MAE criteria, it was possible to identify the best Markov model for each granularity. To check which granularity presents the best Markov model, we used the MAPE criterion, and it was found that the granularity that presented the least error measure was 0.01 granularity. We detected that Markov's 7th order generated better forecasts for the Bitcoin, Ethereum, and Ripple series of categorized returns. For Litecoin, the 9th order model was the most appropriate. These facts characterize the presence of long-range memory in the investigated series. We calculated the support of the transitions that happened most in each chosen model and also verified through the experimental results that the proposed methodology is capable of projecting future perspectives for the return values considering the dependence on discovered memory for cryptocurrencies.

Thus, we extend the existing literature identifying, in addition to the presence of memory, the memory steps present in the series of returns of the main cryptocurrencies in terms of capitalization. The main contributions of this work include a predictive approach not yet used for series of cryptocurrencies, which present behaviour as complex and dynamic as observed in the series of traditional financial markets. As discussed in the categorization time series process, how smaller is the granularity value, better is the precision in the extracted rules. 


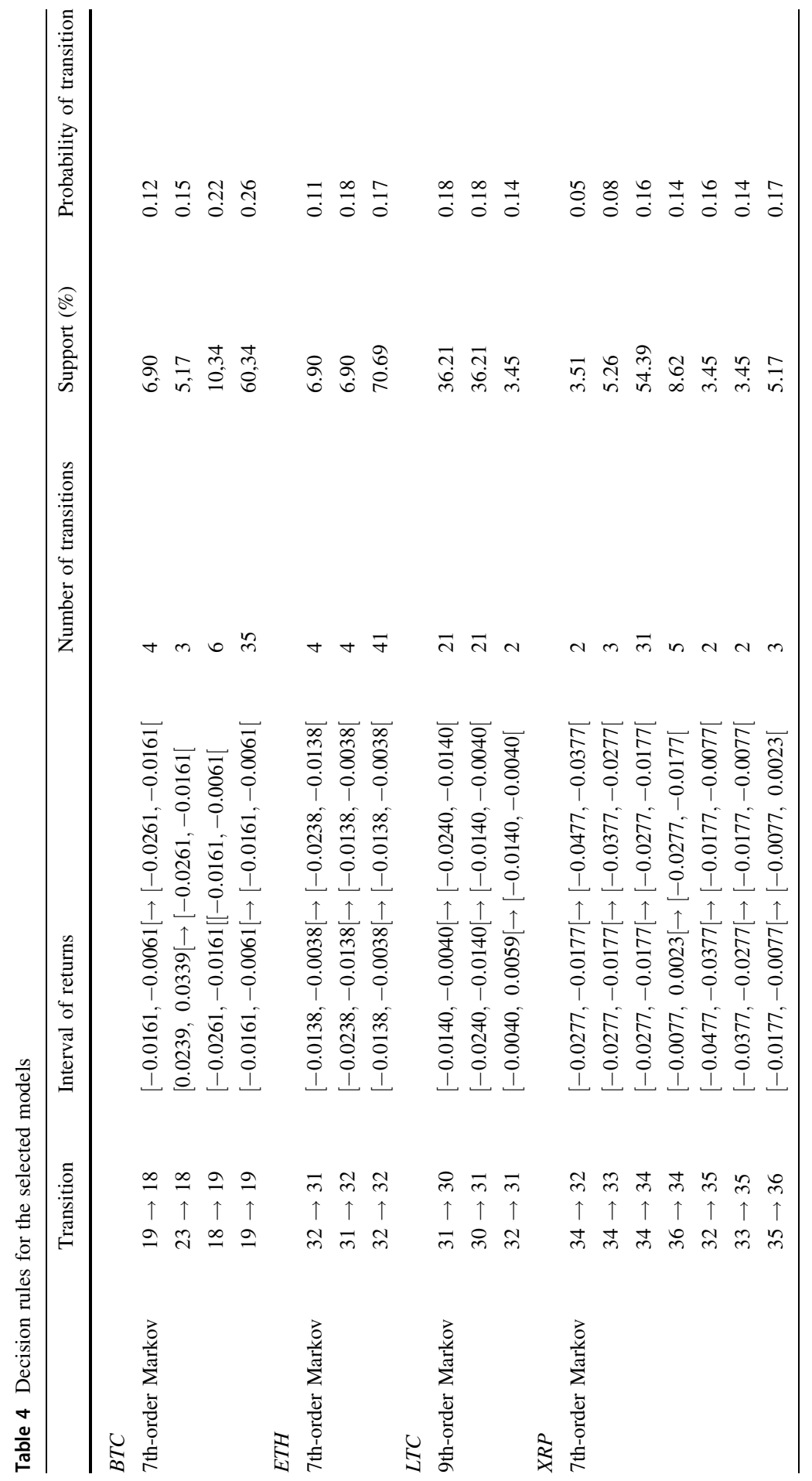


However, if the granularity value is very small, the number of states is enormous. In these conditions, the computational and memory cost of the proposed methodology is high. In general, when the algorithm works with matrices, the memory cost is the product of the number of rows and columns. For the first-order Markov chain, the transition probability matrix is squared, the number of rows is equal to the number of columns. The memory cost is $\Theta\left(n^{2}\right)$, where $n$ is the number of rows (or the number of possible states for the problem aboard here). However, generalizing the memory cost for high-order Markov chains, $m$-order Markov chain memory cost will be $\Theta\left(n^{m+1}\right)$, where $n$ increases with the granularity decrease. In this way, the memory cost was our practical limitation, where it was possible to investigate up to the ten-order Markov chain. The computation cost (the processing cost) increases in the same way as the memory cost multiplied by the number of the time series points $N$, but the memory cost is more critical in the practical sense.

The findings in this work lead us to help investors and policymakers in making investment decisions in the cryptocurrency markets. The results show that the cryptocurrency returns times series have memory, and which is this memory. This information makes it possible to determine better observation windows about the times series data, concentrating the data analysis where it is crucial. In the case of BTC, ETH, and XRP, the relevant observation windows are composed of the last seven lags. For LTC, the previous nine lags. With this analysis, the decision rules (presented in Table 4) can help the investors in the decision make process. Given this better understanding of the cryptocurrency market behavior, investors will obtain better profits and/or minimize their financial losses.

Furthermore, currently, the world suffers from the COVID-19 pandemic. This new world situation can change the behavior of the financial market, in particular the cryptocurrency market. Thus, a new investigation has been started to compare the extracted rules analyzing data before and after the COVID-19 pandemic began. For further research, we will employ other methodologies to categorize the time series data and different forecasting horizons to reduce the error between the future value and the forecasted value. As well as expanding the training series or making a composition of the price value taking into account opening, closing, intraday minimum, and maximum intraday.

Acknowledgements To the Science and Technology Support Foundation of Pernambuco (FACEPE) Brazil, Brazilian National Council for Science and Technological (CNPq), and Coordination for the Improvement of Higher Education Personnel - Brazil (CAPES) - Finance Code 001 by financial support for the development of this research.

Funding Coordination for the Improvement of Higher Education Personnel - Brazil (CAPES) - Finance Code 001, Science and Technology Support Foundation of Pernambuco (FACEPE) Brazil, and Brazilian National Council for Science and Technological (CNPq).

Data Availability Not applicable.

Code Availability Not applicable. 


\section{Declarations}

Conflict of interest The authors declare that they have no conflict of interest.

\section{References}

Almeida, J., Tata, S., Moser, A., \& Smit, V. (2015). Bitcoin prediciton using ANN. Neural Networks, 7, $1-12$.

Altan, A., Karasu, S., \& Bekiros, S. (2019). Digital currency forecasting with chaotic meta-heuristic bioinspired signal processing techniques. Chaos, Solitons \& Fractals, 126, 325-336. https://doi.org/10. 1016/j.chaos.2019.07.011

Antulov-Fantulin, N., Guo, T., \& Lillo, F. (2020). Temporal mixture ensemble models for intraday volume forecasting in cryptocurrency exchange markets. arXiv preprint arXiv:200509356.

Bouri, E., Lau, C. K. M., Lucey, B., \& Roubaud, D. (2019). Trading volume and the predictability of return and volatility in the cryptocurrency market. Finance Research Letters, 29, 340-346. https:// doi.org/10.1016/j.frl.2018.08.015

Bouri, E., Saeed, T., Vo, X. V., \& Roubaud, D. (2021). Quantile connectedness in the cryptocurrency market. Journal of International Financial Markets, Institutions and Money, 71, 101302.

Bouri, E., Vo, X. V., \& Saeed, T. (2021). Return equicorrelation in the cryptocurrency market: Analysis and determinants. Finance Research Letters, 38, 101497.

Caporale, G. M., Gil-Alana, L., \& Plastun, A. (2018). Persistence in the cryptocurrency market. Research in International Business and Finance, 46, 141-148. https://doi.org/10.1016/j.ribaf.2018.01.002

Carvalho, G., \& Moura, A. (2015). Land Cover projection based on Chain Markov and Cellular Automata: Case study of Pampulha-Brazil. In: Proceedings of the International Conference on Changing Cities II: Spatial, Design, Landscape \& Socio-economic Dimensions.

Catania, L., \& Grassi, S. (2017). Modelling crypto-currencies financial time-series. Available at SSRN, 3028486, https://doi.org/10.2139/ssrn.3028486.

Catania, L., Grassi, S., \& Ravazzolo, F. (2019). Forecasting cryptocurrencies under model and parameter instability. International Journal of Forecasting, 35(2), 485-501. https://doi.org/10.1016/j. ijforecast.2018.09.005.

Charfeddine, L., \& Maouchi, Y. (2019). Are shocks on the returns and volatility of cryptocurrencies really persistent? Finance Research Letters, 28, 423-430. https://doi.org/10.1016/j.frl.2018.06.017

Chaudhuri, S., Goswami, S., Das, D., \& Middey, A. (2014). Meta-heuristic ant colony optimization technique to forecast the amount of summer monsoon rainfall: skill comparison with Markov chain model. Theoretical and applied climatology, 116(3-4), 585-595. https://doi.org/10.1007/s00704013-0977-y.

Cheng, Q., Liu, X., \& Zhu, X. (2019). Cryptocurrency momentum effect: DFA and MF-DFA analysis. Physica A: Statistical Mechanics and its Applications, 526, 120847. https://doi.org/10.1016/j.physa. 2019.04.083

Chowdhury, R., Rahman, M.A., Rahman, M.S., \& Mahdy, M. (2019). Predicting and forecasting the price of constituents and index of cryptocurrency using machine learning. arXiv preprint arXiv: 190508444.

Chowdhury, R., Rahman, M. A., Rahman, M. S., \& Mahdy, M. (2020). An approach to predict and forecast the price of constituents and index of cryptocurrency using machine learning. Physica A: Statistical Mechanics and its Applications. https://doi.org/10.1016/j.physa.2020.124569.

CoinMarketCap (2020a) https://coinmarketcap.com/ptbr/all/views/all/ Acessed on 19 May 2020.

CoinMarketCap (2020b) https://coinmarketcap.com/pt-br/ Acessed on 19 May 2020.

da Silva, Jale J., Júnior, S. F. A. X., Xavier, É. F. M., Stošić, T., Stošić, B., \& Ferreira, T. A. E. (2019). Application of Markov chain on daily rainfall data in Paraíba-Brazil from 1995-2015. Acta Scientiarum Technology, 41, e37186-e37186. https://doi.org/10.4025/actascitechnol.v41i1.37186

El Alaoui, M., Bouri, E., \& Roubaud, D. (2019). Bitcoin price-volume: A multifractal cross-correlation approach. Finance Research Letters. https://doi.org/10.1016/j.frl.2018.12.011.

Fakhfekh, M., \& Jeribi, A. (2020). Volatility dynamics of crypto-currencies' returns: Evidence from asymmetric and long memory garch models. Research in International Business and Finance, 51, 101075. https://doi.org/10.1016/j.ribaf.2019.101075 
Ferreira, T. A. E., Vasconcelos, G. C., \& Adeodato, P. J. L. (2008). A new intelligent system methodology for time series forecasting with artificial neural networks. Neural Processing Letters, 28(2), 113-129. https://doi.org/10.1007/s11063-008-9085-x

Fujii, T., Mason, J., Chen, A., Kuhn, P., Woodward, W. A., Tripathy, D., et al. (2019). Prediction of bone metastasis in inflammatory breast cancer using a markov chain model. The Oncologist, 24(10), 1322-1330.

Guo, S., \& Ching, W. K. (2021). High-order markov-switching portfolio selection with capital gain tax. Expert Systems with Applications, 165, 113915. https://doi.org/10.1016/j.eswa.2020.113915.

Guo, Y., Han, S., Shen, C., Li, Y., Yin, X., \& Bai, Y. (2018). An adaptive SVR for high-frequency stock price forecasting. IEEE Access, 6, 11397-11404. https://doi.org/10.1109/ACCESS.2018.2806180.

Han, J., Pei, J., \& Kamber, M. (2011). Data mining: Concepts and techniques. Amsterdam: Elsevier.

Hipp, J., Mangold, C., Güntzer, U., \& Nakhaeizadeh, G. (2002). Efficient rule retrieval and postponed restrict operations for association rule mining. In: Pacific-Asia Conference on Knowledge Discovery and Data Mining, Springer, pp. 52-65.

Hyndman, R. J., \& Koehler, A. B. (2006). Another look at measures of forecast accuracy. International Journal of Forecasting, 22(4), 679-688.

Jarque, CM., \& Bera, AK. (1987). A test for normality of observations and regression residuals. International Statistical Review/Revue Internationale de Statistique pp. 163-172.

Jiang, Y., Nie, H., \& Ruan, W. (2018). Time-varying long-term memory in bitcoin market. Finance Research Letters, 25, 280-284. https://doi.org/10.1016/j.frl.2017.12.009.

Karakoyun, E., \& Cibikdiken, A. (2018). Comparison of arima time series model and lstm deep learning algorithm for bitcoin price forecasting. In: The 13th multidisciplinary academic conference in prague 2018 (the 13th mac 2018), pp. 171-180.

Kemeny, J. G., \& Snell, J. L. (1976). Markov chains. New York: Springer-Verlag.

Kiral, E., \& Uzun, B. (2017). Forecasting Closing returns of Borsa Istanbul index with Markov chain process of the fuzzy states. Journal of Economics Finance and Accounting, 4(1), 15-24. https://doi. org/10.17261/Pressacademia.2017.362

Ky, D. X., \& Tuyen, L. T. (2018). A higher order markov model for time series forecasting. International Journal of Applied Mathematics and Statistics, 57(3), 1-18.

Liu, X., Ren, L., Yuan, F., \& Yang, B. (2009). Meteorological drought forecasting using Markov Chain model. In: 2009 International Conference on Environmental Science and Information Application Technology, IEEE, 2, 23-26. https://doi.org/10.1109/ESIAT.2009.19.

Mata, J., Alvarez, JL., \& Riquelme, JC. (2002). Discovering numeric association rules via evolutionary algorithm. In: Pacific-Asia conference on knowledge discovery and data mining, Springer, pp. 40-51.

Mensi, W., Lee, Y. J., Al-Yahyaee, K. H., Sensoy, A., \& Yoon, S. M. (2019). Intraday downward/upward multifractality and long memory in Bitcoin and Ethereum markets: An asymmetric multifractal detrended fluctuation analysis. Finance Research Letters, 31, 19-25. https://doi.org/10.1016/j.frl. 2019.03.029

Naeem, M. A., Bouri, E., Peng, Z., Shahzad, S. J. H., \& Vo, X. V. (2021). Asymmetric efficiency of cryptocurrencies during covid19. Physica A: Statistical Mechanics and its Applications, 565, 125562.

Papaefthymiou, G., \& Klockl, B. (2008). MCMC for wind power simulation. IEEE Transactions on Energy Conversion, 23(1), 234-240. https://doi.org/10.1109/TEC.2007.914174

Peng, Y., Albuquerque, P. H. M., de Sá, J. M. C., Padula, A. J. A., \& Montenegro, M. R. (2018). The best of two worlds: Forecasting high frequency volatility for cryptocurrencies and traditional currencies with Support Vector Regression. Expert Systems with Applications, 97, 177-192. https://doi.org/10. 1016/j.eswa.2017.12.004

Phillip, A., Chan, J., \& Peiris, S. (2019). On long memory effects in the volatility measure of cryptocurrencies. Finance Research Letters, 28, 95-100. https://doi.org/10.1016/j.frl.2018.04.003

Rambaccussing, D., \& Mazibas, M. (2020). True versus spurious long memory in cryptocurrencies. Journal of Risk and Financial Management, 13(9), 186. https://doi.org/10.3390/jrfm13090186

Rezaeianzadeh, M., Stein, A., \& Cox, J. P. (2016). Drought forecasting using Markov chain model and artificial neural networks. Water Resources Management, 30(7), 2245-2259. https://doi.org/10. 1007/s11269-016-1283-0.

Sahin, A. D., \& Sen, Z. (2001). First-order Markov chain approach to wind speed modelling. Journal of Wind Engineering and Industrial Aerodynamics, 89(3-4), 263-269. https://doi.org/10.1016/S01676105(00)00081-7 
Schinckus, C., Nguyen, C. P., \& Ling, F. C. H. (2020). Crypto-currencies trading and energy consumption. International Journal of Energy Economics and Policy, 10(3), 355.

Schinckus, C., Duy, D. P. T., \& Canh, N. P. (2021). Interdependences between cryptocurrencies: A network analysis from 2013 to 2018. Journal of Interdisciplinary Economics, 33(2), 190-199.

Shamshad, A., Bawadi, M., Hussin, W. W., Majid, T., \& Sanusi, S. (2005). First and second order Markov chain models for synthetic generation of wind speed time series. Energy, 30(5), 693-708.

Soloviev, V., Saptsin, V., \& Chabanenko, D. (2011). Markov chains application to the financial-economic time series prediction. arXiv preprint arXiv: 11115254 .

Song, J. Y., Chang, W., \& Song, J. W. (2019). Cluster analysis on the structure of the cryptocurrency market via Bitcoin-Ethereum filtering. Physica A: Statistical Mechanics and its Applications. https:// doi.org/10.1016/j.physa.2019.121339.

Song, Z., Jiang, Y., \& Zhang, Z. (2014). Short-term wind speed forecasting with Markov-switching model. Applied Energy, 130, 103-112. https://doi.org/10.1016/j.apenergy.2014.05.026

Soylu, P. K., Okur, M., Çatıkkaş, Ö., \& Altintig, Z. A. (2020). Long memory in the volatility of selected cryptocurrencies: Bitcoin, Ethereum and Ripple. Journal of Risk and Financial Management, 13(6), 107. https://doi.org/10.3390/jrfm13060107

Stosic, D., Stosic, D., Ludermir, T. B., \& Stosic, T. (2019). Multifractal behavior of price and volume changes in the cryptocurrency market. Physica A: Statistical Mechanics and Its Applications, 520, 54-61. https://doi.org/10.1016/j.physa.2018.12.038

Sun, X., Liu, M., \& Sima, Z. (2020). A novel cryptocurrency price trend forecasting model based on LightGBM. Finance Research Letters. https://doi.org/10.1016/j.frl.2018.12.032.

Svoboda, M., \& Lukas, L. (2012). Application of Markov chain analysis to trend prediction of stock indices. In: Proceedings of 30th international conference mathematical methods in economics. Karviná: Silesian University, School of Business Administration, pp. 848-853.

Takaishi, T. (2018). Statistical properties and multifractality of Bitcoin. Physica A: Statistical Mechanics and its Applications, 506, 507-519. https://doi.org/10.1016/j.physa.2018.04.046

Tiwari, A. K., Jana, R. K., Das, D., \& Roubaud, D. (2018). Informational efficiency of bitcoin-an extension. Economics Letters, 163, 106-109. https://doi.org/10.1016/j.econlet.2017.12.006

Velankar, S., Valecha, S., \& Maji, S. (2018). Bitcoin price prediction using machine learning. In: 2018 20th International Conference on Advanced Communication Technology (ICACT), IEEE, pp. 144-147.

Wu, CH., Lu, CC., Ma, YF., \& Lu, RS. (2018). A new forecasting framework for bitcoin price with lstm. In: 2018 IEEE International Conference on Data Mining Workshops (ICDMW), IEEE, pp. 168-175, https://doi.org/10.1109/ICDMW.2018.00032.

Yi, S., Xu, Z., \& Wang, G. J. (2018). Volatility connectedness in the cryptocurrency market: Is Bitcoin a dominant cryptocurrency? International Review of Financial Analysis, 60, 98-114. https://doi.org/ 10.1016/j.irfa.2018.08.012

Zhan-Li, M., \& Jin-Hua, S. (2011). Application of Grey-Markov model in forecasting fire accidents. Procedia Engineering, 11, 314-318. https://doi.org/10.1016/j.proeng.2011.04.663.

Zhang, W., Wang, P., Li, X., \& Shen, D. (2018). Some stylized facts of the cryptocurrency market. Applied Economics, 50(55), 5950-5965.

Publisher's Note Springer Nature remains neutral with regard to jurisdictional claims in published maps and institutional affiliations. 